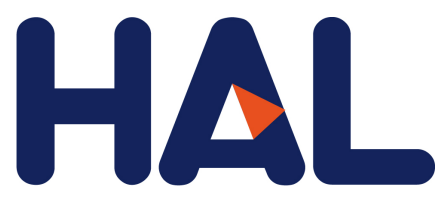

archives-ouvertes

\title{
All-dielectric bulk isotropic double-negative metamaterials
}

\author{
Alexander Zharov, Vanessa Fierro, Alain Celzard
}

\section{To cite this version:}

Alexander Zharov, Vanessa Fierro, Alain Celzard. All-dielectric bulk isotropic double-negative metamaterials. Journal of the Optical Society of America B, Optical Society of America, 2021, 38 (1), pp.159. 10.1364/JOSAB.408571 . hal-03406320

\section{HAL Id: hal-03406320 \\ https://hal.univ-lorraine.fr/hal-03406320}

Submitted on 27 Oct 2021

HAL is a multi-disciplinary open access archive for the deposit and dissemination of scientific research documents, whether they are published or not. The documents may come from teaching and research institutions in France or abroad, or from public or private research centers.
L'archive ouverte pluridisciplinaire HAL, est destinée au dépôt et à la diffusion de documents scientifiques de niveau recherche, publiés ou non, émanant des établissements d'enseignement et de recherche français ou étrangers, des laboratoires publics ou privés. 


\title{
All-dielectric bulk isotropic double-negative metamaterials
}

\author{
Alexander Zharov ${ }^{1,2,3}$, Vanessa Fierro ${ }^{1}$, Alain Celzard ${ }^{1,4}$ \\ ${ }^{1}$ Université de Lorraine, CNRS, IJL, F-88000 Epinal, France \\ ${ }^{2}$ Institute for Physics of Microstructures, Russian Academy of Sciences, Nizhny Novgorod 603950, \\ Russia \\ 3 alexander.zharov@univ-lorraine.fr \\ 4 alain.celzard@univ-lorraine.fr
}

\begin{abstract}
We propose the design of all-dielectric isotropic micro- or nanoparticles which, when used as structural elements of a metamaterial, can simultaneously provide negative effective electric permittivity and negative magnetic permeability. The particles comprise three dielectric layers with specially adjusted parameters, which ensure simultaneous excitation of the electric and magnetic Mie dipole modes. The layer parameters were found by the Mie scattering analysis and then verified by the simulations, which confirmed the double-negative behavior of the metamaterial at up to visible light frequencies. The isotropy of such particles suggests the possibility of using them in a double-negative liquid metamaterial.
\end{abstract}

\section{Introduction}

In recent years, metamaterial studies have become a distinct branch of modern electrodynamics. Metamaterials are artificial media with specially designed structural subwavelength elements, called meta-atoms, which allow obtaining macroscopic electromagnetic properties unattainable in natural materials. Since the theoretical predictions [1-3] and the first experimental observations [4,5], many different metamaterials have been developed, such as linear and nonlinear double-negative metamaterials (DNMs) for the wavelength range from microwaves to visible light [6-12], hyperbolic materials [13-15], near-zero-index metamaterials [16,17], and liquid metamaterials [18-20], among others. Another particularity of metamaterials is their extremely high potential tunability, which can be achieved, for example, by immersing them in a nematic liquid crystal, allowing the use of various strategies, including those based on temperature, the application of external voltages or magnetic fields, and all-optical methods that make use of the highly nonlinear responses of materials [21-23]. Their unique properties have motivated applications such as invisibility cloaking [24,25], superlensing [26,27], transformation optics [28,29], phase and polarization manipulation [30,31], and enhancement of chirality and optical activity [32,33].

In general, DNMs, i.e., metamaterials with simultaneously negative permittivity and negative permeability, are based on metallic components [34-38]. For instance, in microwave DNMs, split-ring resonators are generally used, while in optical or infrared (IR) DNMs, fishnet structures are common. The main limitation in the use of metallic constituents is the unavoidable occurrence of Ohmic damping, which results in strong absorption at higher frequencies. In addition, as the plasmon resonance frequency is approached, the magnetic response weakens, further limiting the use of metal-based DNMs at high frequencies [39]. To overcome these issues, high-index dielectric particles have been suggested as building blocks of metamaterials [40]. Indeed, as Mie has shown [41], such particles may have both electric and magnetic resonant responses, which were analyzed in $[42,43]$. However, in homogeneous spherical particles, these resonances appear at different frequencies. To match both magnetic and electric responses at the same frequency, several methods have been proposed, such as 
metallic coatings for an additional plasmonic response [44-46], non-spherical particles [47], sub-lattices of particles of different sizes [48], and dielectric-coated metal particles with perfect conductor-like response [49]. Moreover, all-dielectric core-shell particles were suggested for double-negative response at microwave frequencies [50]. However, the design proposed in [50] relies on the exceptionally high values of the refractive index, which are not available in the higher-frequency regions.

In this paper, we propose theoretical studies of an all-dielectric meta-atom design for a DNM, which may provide low loss and high scalability. By using three-layer radially inhomogeneous dielectric spherical structures, one may achieve a simultaneous excitation of electric and magnetic dipole Mie resonances at up to visible light frequencies. As a result, an array of such particles at a sufficient concentration shall simultaneously have negative effective permittivity and negative permeability, i.e., shall behave as a DNM. Moreover, due to isotropy, an ensemble of the proposed meta-atoms does not need any special arrangement [51] to provide a double-negative response. Thus, as a particular realization, such particles can be suspended in a fluid, forming a liquid DNM, which presents even more possibilities for controlling electromagnetic waves.

\section{Dielectric spherical particles: electric and magnetic responses}

The original Mie theory describes the scattering of a plane electromagnetic wave over a spherical homogeneous dielectric particle. It predicts the existence of both magnetic and electric dipole resonances in such a particle. However, these resonances are always separated. Namely, the fundamental magnetic dipole resonance has a lower frequency than the fundamental electric resonance. Therefore, to control the relative positions of the resonances, structures that are more complex have to be considered.

The Mie theory can be generalized for the case of a layered dielectric spherical particle with different permittivity values assigned to each layer [52,53]. The analytical solution is obtained by expanding the fields in vector spherical harmonics, and the scattered field is entirely characterized by the scattering coefficients $a_{n}$ and $b_{n}$, which represent electric and magnetic $2^{n}$-pole mode amplitudes, respectively. In a sphere, each layer can be characterized by its relative refractive index $m_{i}=n_{i} / n_{h}$ and the dimensionless size parameter $x_{i}=$ $2 \pi n_{h} r_{i} / \lambda=n_{h} r_{i} k_{0}$. Here, $n_{i}$ and $n_{h}$ are the refractive indices of the $i^{\text {th }}$ layer and the surrounding medium (host medium), respectively; $r_{i}$ is the radius of the $i^{\text {th }}$ layer; $\lambda$ is the freespace incident radiation wavelength; and $k_{0}=\omega / c$ is the free-space wavenumber, in which $\omega$ is the radiation frequency and $c$ is the speed of light. Thus, for an $L$-layer sphere, the scattering coefficients can be expressed as:

$$
\begin{gathered}
a_{n}=\frac{\left[H_{n}^{a}\left(m_{L} x_{L}\right) / m_{L}+n / x_{L}\right] \psi_{n}\left(x_{L}\right)-\psi_{n-1}\left(x_{L}\right)}{\left[H_{n}^{a}\left(m_{L} x_{L}\right) / m_{L}+n / x_{L}\right] \zeta_{n}\left(x_{L}\right)-\zeta_{n-1}\left(x_{L}\right),} \\
b_{n}=\frac{\left[H_{n}^{b}\left(m_{L} x_{L}\right) / m_{L}+n / x_{L}\right] \psi_{n}\left(x_{L}\right)-\psi_{n-1}\left(x_{L}\right)}{\left[H_{n}^{b}\left(m_{L} x_{L}\right) / m_{L}+n / x_{L}\right] \zeta_{n}\left(x_{L}\right)-\zeta_{n-1}\left(x_{L}\right)},
\end{gathered}
$$

where $\psi_{n}(z)=z j_{n}(z)$ and $\zeta_{n}(z)=z h_{n}^{(1)}(z)$ are the Riccati-Bessel functions expressed through the spherical Bessel functions $j_{n}(z)$ and $h_{n}^{(1)}(z) . H_{n}^{a}$ and $H_{n}^{b}$ can be found using the following recursive expressions [53]:

$$
\begin{gathered}
H_{n}^{a}\left(m_{1} x_{1}\right)=D_{n}^{(1)}\left(m_{1} x_{1}\right) \\
H_{n}^{a}\left(m_{i} x_{i}\right)=\frac{G_{2} D_{n}^{(1)}\left(m_{i} x_{i}\right)-Q_{n}^{(i)} G_{1} D_{n}^{(3)}\left(m_{i} x_{i}\right)}{G_{2}-Q_{n}^{(i)} G_{1}}, \\
H_{n}^{b}\left(m_{1} x_{1}\right)=D_{n}^{(1)}\left(m_{1} x_{1}\right) \\
H_{n}^{b}\left(m_{i} x_{i}\right)=\frac{\tilde{G}_{2} D_{n}^{(1)}\left(m_{i} x_{i}\right)-Q_{n}^{(i)} \tilde{G}_{1} D_{n}^{(3)}\left(m_{i} x_{i}\right)}{\tilde{G}_{2}-Q_{n}^{(i)} \tilde{G}_{1}},
\end{gathered}
$$




$$
\begin{gathered}
D_{n}^{(1)}(z)=\frac{\Psi_{n}^{\prime}(z)}{\Psi_{n}(z)^{\prime}} \\
D_{n}^{(3)}(z)=\frac{\zeta_{n}^{\prime}(z)}{\zeta_{n}(z)}, \\
Q_{n}^{(i)}=\frac{\Psi_{n}\left(m_{i} x_{i-1}\right) / \zeta_{n}\left(m_{i} x_{i-1}\right)}{\Psi_{n}\left(m_{i} x_{i}\right) / \zeta_{n}\left(m_{i} x_{i}\right)}, \\
G_{1}=m_{i} H_{n}^{a}\left(m_{i-1} x_{i-1}\right)-m_{i-1} D_{n}^{(1)}\left(m_{i} x_{i-1}\right), \\
G_{2}=m_{i} H_{n}^{a}\left(m_{i-1} x_{i-1}\right)-m_{i-1} D_{n}^{(3)}\left(m_{i} x_{i-1}\right), \\
\tilde{G}_{1}=m_{i-1} H_{n}^{b}\left(m_{i-1} x_{i-1}\right)-m_{i} D_{n}^{(1)}\left(m_{i} x_{i-1}\right), \\
\tilde{G}_{2}=m_{i-1} H_{n}^{b}\left(m_{i-1} x_{i-1}\right)-m_{i} D_{n}^{(3)}\left(m_{i} x_{i-1}\right),
\end{gathered}
$$

where $i=2 . . L$ and the prime represents a derivative with respect to the argument. The layered structure of a particle is accounted for by the recursive expressions (2). Namely, all information about the internal structure of the particle is contained in the coefficients $H_{n}^{a}$ and $H_{n}^{b}$, which are calculated layer-by-layer from the center to the surface of the particle. When the particle is small compared to the wavelength, only the dipole terms can be taken into account, while the higher-order multipole terms can be neglected. As the scattered electric and magnetic dipole fields are proportional to $a_{1}$ and $b_{1}$, respectively, the corresponding polarizabilities $\alpha_{e}$ and $\alpha_{m}$ can be calculated as follows [54]:

$$
\begin{aligned}
& \alpha_{e}=\frac{6 \pi i a_{1}}{\left(n_{h} k_{0}\right)^{3}} \\
& \alpha_{m}=\frac{6 \pi i b_{1}}{\left(n_{h} k_{0}\right)^{3}}
\end{aligned}
$$

To estimate the macroscopic electromagnetic behavior of a composite based on such particles distributed in a host medium, the Maxwell Garnett (MG) effective medium approximation can be applied [55]. In this context, the relative material parameters of the composite can be calculated as:

$$
\begin{gathered}
\epsilon_{e f f}=\epsilon_{h}\left(1+\frac{3 i f a_{1}}{2 / 3 x_{L}^{3}-i f a_{1}}\right) \\
\mu_{e f f}=1+\frac{3 i f b_{1}}{2 / 3 x_{L}^{3}-i f b_{1}},
\end{gathered}
$$

where $f=V N_{p}$ is the filling fraction of the composite; $V=4 \pi r_{L}^{3} / 3$ is the particle volume and $N_{p}$ is the concentration of particles. Expressions (4) and (5) imply resonant behaviors of electric permittivity and magnetic permeability. Thus, at sufficiently high values of the filling fraction, either of the two indices can become negative. At the same time, the resonance frequencies of $\epsilon_{e f f}$ and $\mu_{\text {eff }}$ depend on the specific composition of the particles and are defined by dipole scattering coefficients $a_{1}$ and $b_{1}$. For instance, as is well known in a homogeneous particle $(L=1)$, the fundamental magnetic resonance has a frequency essentially lower than that of the fundamental electric one. However, by varying the design of a layered particle, it is possible to change the relative positions of the magnetic and electric resonances. Hence, expressions (4) and (5) may be used to detect combinations of parameters that would simultaneously provide negative electric permittivity and negative magnetic permeability. 
To manipulate the relative positions of electric and magnetic resonances, we considered three-layer particles $(L=3)$ with different refraction coefficients assigned to each layer. The detailed design of this structure is shown in Fig. 1. We examined two frequency ranges, terahertz $(\mathrm{THz})$ and visible light, to demonstrate the scalability of the system. Within the considered frequency ranges, we neglected the frequency dispersion of the layer materials and assumed the refraction indices constant. For each frequency range, we set the core radius $r_{1}$ and the refraction index $m_{1}$ and varied the layer thicknesses $h_{1}$ and $h_{2}$ as well as the layer refractive indices $m_{2}$ and $m_{3} ; r_{2}=r_{1}+h_{1}$ and $r_{3} \equiv r_{L}=r_{1}+h_{1}+h_{2}$.
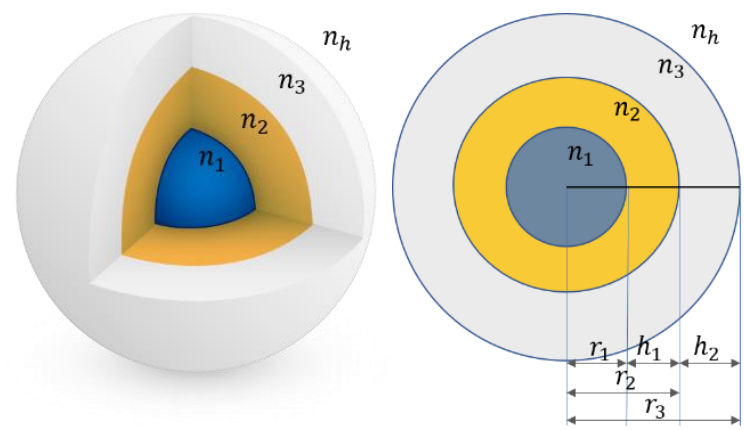

Fig. 1. Structure of a dielectric three-layer particle. The radii of the layers are $r_{1}, r_{2}=r_{1}+h_{1}$, and $r_{3}=r_{1}+h_{1}+$ $h_{2}$. The refractive indices of the layers are $n_{1}, n_{2}$, and $n_{3}$. The particle is surrounded by a host medium of refractive index $n_{h}$.

First, we optimized the particle design for the $\mathrm{THz}$ frequency range. In $\mathrm{THz}$, materials with extremely high permittivity exist. For example, processed $\mathrm{SrTiO}_{3}$ with a relative permittivity as high as $\sim 340$ at $0.3-0.9 \mathrm{THz}$ has been reported [56]. Nevertheless, we set the maximum relative permittivity to $\epsilon_{\max }=100$, which corresponds to a refractive index of $n_{\max }=$ $\sqrt{\epsilon_{\max }}=10$. The relative permittivity of the host medium was set to 1 . The results of the optimization are shown in Fig. 2. To estimate the left-handed performance of a material, we used the value of $\Psi$, which represents a measure of the overlap of the negative real parts of effective electric permittivity and magnetic permeability and can be calculated as follows:

$$
\begin{gathered}
\Psi=-\frac{1}{2} \int(\psi(\omega)-|\psi(\omega)|) d \omega, \\
\psi(\omega)=\operatorname{Re}(\sqrt{\operatorname{Re} \epsilon(\omega)} \sqrt{\operatorname{Re} \mu(\omega)})
\end{gathered}
$$

where $\epsilon(\omega)$ and $\mu(\omega)$ are the effective permittivity and permeability calculated using the MG approximation and $\omega$ is the frequency. It can be seen that the function $\Psi$ is non-zero only when there is a frequency region with simultaneously negative permittivity and permeability. In addition, the larger this region is, the larger this function becomes. To illustrate this, let us consider first the function $\psi(\omega)=\operatorname{Re}(\sqrt{\operatorname{Re} \epsilon(\omega) \sqrt{\operatorname{Re} \mu(\omega)}})$, Eq. (7). Clearly, $\psi(\omega)$ is positive when $\operatorname{Re} \epsilon(\omega)$ and $\operatorname{Re} \mu(\omega)$ are both positive, negative when $\operatorname{Re} \epsilon(\omega)$ and $\operatorname{Re} \mu(\omega)$ are both negative, and zero when $\operatorname{Re} \epsilon(\omega)$ and $\operatorname{Re} \mu(\omega)$ have different signs. Then, the function $\Psi$, Eq. (6), which integrates the negative values of $\psi(\omega)$ over all the frequencies, thus represents some measure of the frequency region of simultaneously negative permittivity and permeability. Although the function $\Psi$ does not have a well-defined physical meaning, it gives an idea of whether or not the frequency region of double-negative behavior exists. 


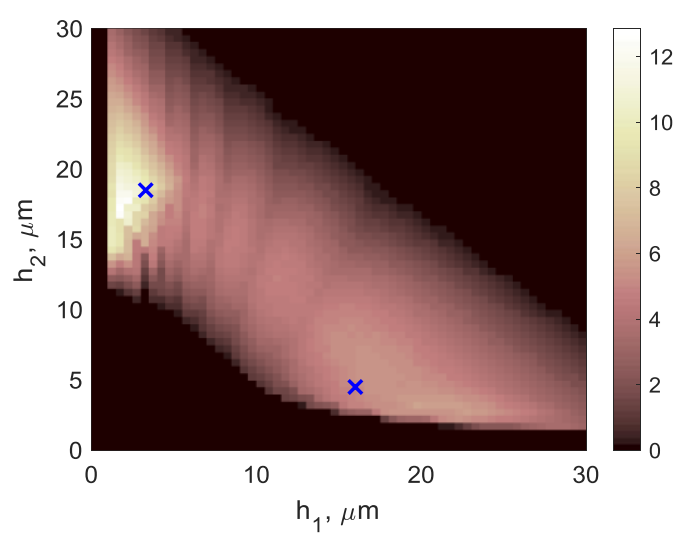

Fig. 2. Optimization of the particle design for negative refraction in the THz band. The color scale represents $\Psi$ (Eqs. $(6,7)) ; x$ and $y$ axes correspond to the layer thicknesses $h_{1}$ and $h_{2}$. The core radius was set to $20 \mu \mathrm{m}$; the core relative permittivity was set to 10 . The layer relative permittivity values were varied from 1 to 10 ; the relative permittivity of the host medium was set to 1 ; the filling fraction was 0.52 . The blue crosses represent two sets of particle parameter used for the simulations.

Second, we repeated the procedure for the visible frequency range. The main difference compared to $\mathrm{THz}$ is that in the optical frequency range, the maximum value of material permittivity is significantly lower. Namely, silicon has a relative permittivity value of $\sim 11$, germanium has relative permittivity of $\sim 16$, and the relative permittivity of tellurium is as high as $\sim 25$. Therefore, in our calculations, the maximum refractive index was limited to 5 , which correspond to Te. The results of the optimization are presented in Fig. 3. Compared to the $\mathrm{THz}$ range, in the visible we could not simultaneously find negative permittivity and negative permeability for filling fractions lower than $\sim 0.68$.

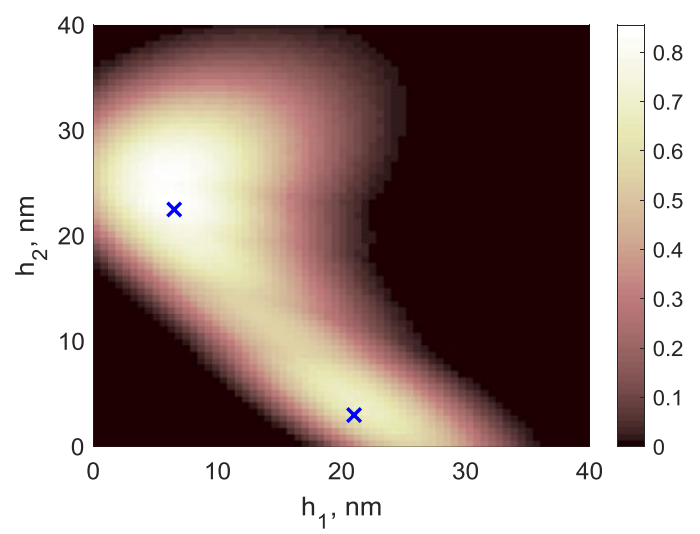

Fig. 3. Same as Fig. 2, but for the visible light band, using the following parameters. The core radius was set to 30 $\mathrm{nm}$; the core relative permittivity was set to 5. The layer relative permittivity values were varied from 1 to 5; the relative permittivity of the host medium was set to 1 ; the filling fraction was 0.685 . The blue crosses represent two sets of particle parameters used for the simulations.

Figures 2 and 3 show that the proposed particle design offers some flexibility with respect to variations in particle parameters. Namely, the parameters corresponding to the doublenegative behavior on the parametric planes in Figs. 2 and 3 form a continuous set. Therefore, a small deviation from a chosen point does not cause the double-negative behavior to break, as long as the new parameters are always located in the areas highlighted in Figs. 2 and 3.

It should be noted that, strictly speaking, the validity conditions of the MG approximation are violated for the sets of parameter representing the optimal designs. Indeed, in these cases, the particles cannot be treated as point dipoles and the filling fractions reach values near those of 
a close-packing structure, which is unacceptable for a thorough treatment within the limits of the MG approximation. However, the obtained results may be considered as possible designs, which should be subject to additional verification. In the next section, we present the results of full-wave simulation of the proposed material to support the analytical considerations. The experimental verification, however, is well beyond the scope of the present theoretical study, which should be seen as preliminary work intended to encourage future experimental work.

\section{Simulation results and discussion}

In accordance with the above reasoning, several specific particle designs were chosen as illustrative examples. Again, we considered particles for $\mathrm{THz}$ and visible-light applications. The simulations were performed using COMSOL Multiphysics. The effective material parameters were calculated with the field-averaging method [57].

First, we examined the THz-optimized designs. Figure 4 shows the simulated effective relative electric permittivity and magnetic permeability, as well as the effective refractive index, of a medium composed of layered dielectric particles as a function of frequency. The parameters of the particles were set as follows: (a,c) $r_{1}=20.0 \mu \mathrm{m} ; h_{1}=3.28 \mu \mathrm{m} ; h_{2}=$ $18.5 \mu \mathrm{m} ; m_{1}=10+0.01 i ; m_{2}=1.5+0.01 i ; m_{3}=6+0.01 i$ and $(\mathrm{b}, \mathrm{d}) \quad r_{1}=20.0 \mu \mathrm{m}$; $h_{1}=16.0 \mu \mathrm{m} ; h_{2}=4.5 \mu \mathrm{m} ; m_{1}=10+0.01 i ; m_{2}=3+0.01 i ; m_{3}=10+0.01 i$. The figure of merit (FOM), i.e., the ratio of the real negative part of the refractive index to its imaginary part, was found to be as high as $\sim 3.16$. The refractive index of the host medium was set to 1 , and the filling fraction was $\sim 0.30$. In Fig. 4 , it is clearly seen that there are regions of simultaneously and markedly negative material indices. This indicates that in these regions, such materials must exhibit negative refraction.
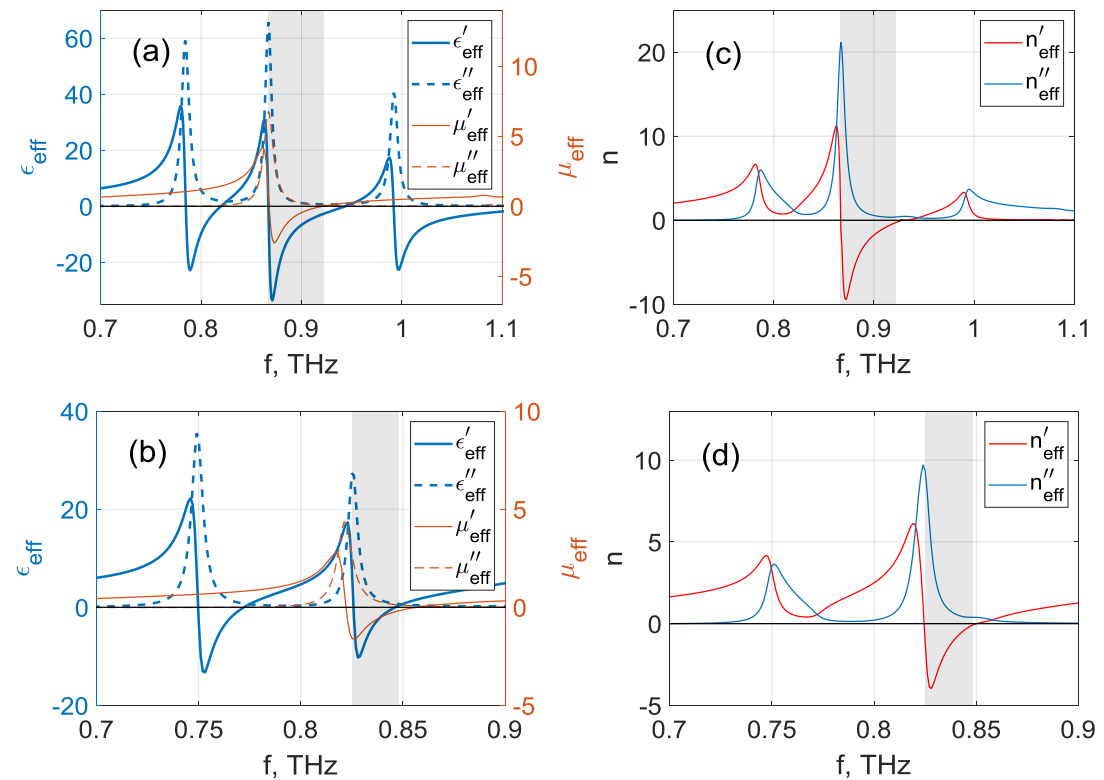

Fig. 4. Relative electric permittivity and magnetic permeability (a,b) and effective refractive index (c,d) of a medium composed of three-layer dielectric particles in the THz frequency band. In (a) and (b), the thick blue lines represent real (solid) and imaginary (dashed) parts of complex electric permittivity, $\epsilon_{e f f}=\epsilon_{e f f}^{\prime}+i \epsilon^{\prime \prime}{ }_{e f f}$; the thin brown lines represent real (solid) and imaginary (dashed) parts of complex magnetic permeability, $\mu_{e f f}=\mu_{e f f}^{\prime}+i \mu^{\prime \prime}{ }_{e f f}$. In (c) and (d), the red lines represent the real part of the refractive index, and the blue line represents the imaginary part of the refractive index. The shaded area represents the frequency range where the real parts of both the effective permittivity and permeability, as well as the refractive index, are negative. The parameters of the particle were as follows: (a,c) $r_{1}=20.0 \mu \mathrm{m} ; h_{1}=3.28 \mu \mathrm{m} ; h_{2}=18.5 \mu \mathrm{m} ; m_{1}=10+0.01 i ; m_{2}=1.5+0.01 i ; m_{3}=6+0.01 i$ 
and $(\mathrm{b}, \mathrm{d}) \quad r_{1}=20.0 \mu \mathrm{m} ; h_{1}=16.0 \mu \mathrm{m} ; h_{2}=4.5 \mu \mathrm{m} ; m_{1}=10+0.01 i ; m_{2}=3+0.01 i ; m_{3}=10+0.01 i$. The refractive index of the host material was set to 1 ; the filling fraction was $\sim 0.30$.

Figure 5 shows the effective relative electric permittivity, magnetic permeability and refractive index of a medium composed of two different particle designs optimized for the visible-light frequency band. The parameters of the particles were set as follows: (a,c) $r_{1}=30.0 \mathrm{~nm} ; h_{1}=6.5 \mathrm{~nm} ; h_{2}=25.5 \mathrm{~nm} ; m_{1}=5+0.1 i ; m_{2}=4.5+0.1 i ; m_{3}=3.8+$ $0.1 i$ and $(\mathrm{b}, \mathrm{d}) r_{1}=30 \mathrm{~nm} ; h_{1}=21 \mathrm{~nm} ; h_{2}=3 \mathrm{~nm} ; m_{1}=5+0.1 i ; m_{2}=3.5+0.1 i$; $m_{3}=5+0.1 i$. The FOM was found to be as high as $\sim 3.14$. The refractive index of the host medium was set to 1 ; the filling fraction was $\sim 0.39$. According to refractive index databases, the desired properties of the materials discussed here might be obtained from particles with, for instance, a tellurium core and outer shells made of different metallic tellurides. Again, in both cases, there are distinct regions of simultaneously negative permittivity and negative permeability. It should be noted that the filling fraction in the simulation was significantly lower than the fraction expected to be necessary for the double-negative response by the MG approximation.
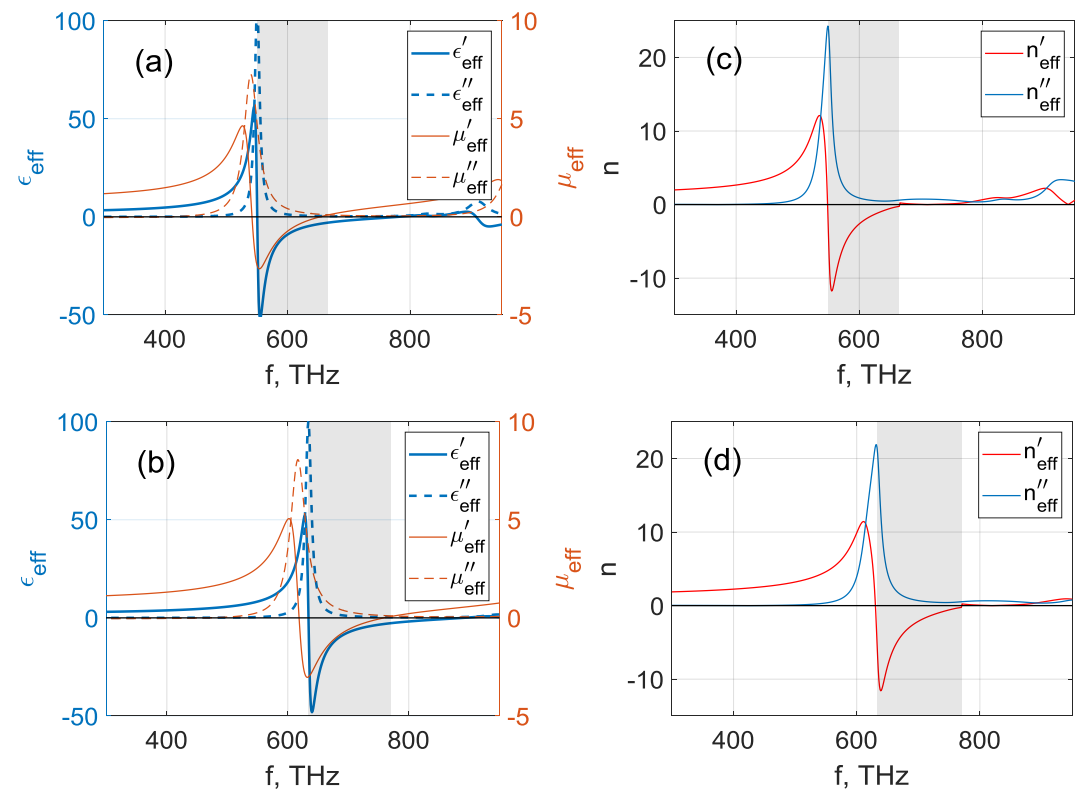

Fig. 5. Same as Fig. 4 but for the visible-light frequency band, using the following particle parameters: (a,c) $r_{1}=$ $30.0 \mathrm{~nm} ; h_{1}=6.5 \mathrm{~nm} ; h_{2}=25.5 \mathrm{~nm} ; m_{1}=5+0.1 i ; m_{2}=4.5+0.1 i ; m_{3}=3.8+0.1 i$ and (b,d) $r_{1}=30 \mathrm{~nm}$; $h_{1}=21 \mathrm{~nm} ; h_{2}=3 \mathrm{~nm} ; m_{1}=5+0.1 i ; m_{2}=3.5+0.1 i ; m_{3}=5+0.1 i$. The refractive index of the host material was set to 1 ; the filling fraction was $\sim 0.39$.

As the proposed metamaterial uses resonance effects, there is an intrinsic amplification of the losses at the resonance, which corresponds to the peak of the imaginary part of the effective refractive index. It is evident that such a loss amplification reduces the metamaterial performance and may compromise the advantages over metallic metamaterials. As mentioned above, the FOM of the proposed metamaterial is approximately 3.15 in both the $\mathrm{THz}$ and visible ranges. For comparison, the following values of FOM are given in the literature: 0.6 at $3.65 \mathrm{THz}$ [45]; 10.9 at $1 \mathrm{THz}$ [35]; 23 at $1.3 \mathrm{THz}$ [37]; 5 at $1 \mathrm{THz}$ [38] in the $\mathrm{THz}$ range and 0.2 at $775 \mathrm{THz}$ [46]; 0.3 at $176 \mathrm{THz}$ [34]; 0.3 at $517 \mathrm{THz}$ [36] in the visible range. Therefore, it is clear that the use of the proposed metamaterial is reasonable in the higher-frequency ranges, e.g. in the visible range.

In order to analyze the influence of changes in particle parameter on the metamaterial performance, several simulations with various parameters were carried out. Figure 6 shows 
the frequency dependencies of the effective permittivity and permeability for three sets of parameters in the $\mathrm{THz}$ and visible bands. It can be seen that slight deviations of the metaatom parameters do not jeopardize the double-negative behavior.
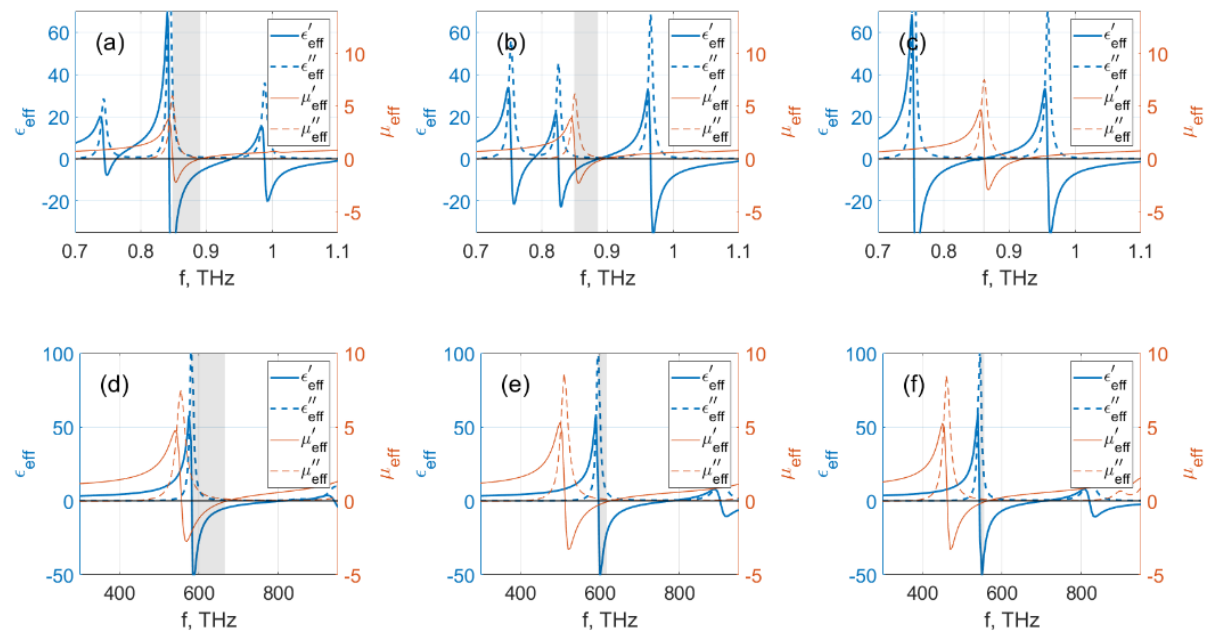

Fig. 6. Influence of changes in the particle parameters on the double-negative behavior of the metamaterial in $\mathrm{THz}$ $(\mathrm{a}, \mathrm{b}, \mathrm{c})$ and visible $(\mathrm{d}, \mathrm{e}, \mathrm{f})$ ranges. The area of simultaneously negative effective permittivity and permeability is highlighted in gray. The parameters of particles are as follows: (a) $r_{1}=20.0 \mu \mathrm{m} ; h_{1}=3.1 \mu \mathrm{m} ; h_{2}=18.8 \mu \mathrm{m}$; $m_{1}=10+0.01 i ; m_{2}=1.4+0.01 i ; m_{3}=6.5+0.01 i ;$ (b) $r_{1}=20.0 \mu \mathrm{m} ; h_{1}=3.5 \mu \mathrm{m} ; h_{2}=19.0 \mu \mathrm{m} ; m_{1}=$ $10+0.01 i ; m_{2}=1.8+0.01 i ; m_{3}=6.2+0.01 i ;$ (c) $r_{1}=20.0 \mu \mathrm{m} ; h_{1}=3.0 \mu \mathrm{m} ; h_{2}=20.5 \mu \mathrm{m} ; m_{1}=10+$ $0.01 i ; m_{2}=2+0.01 i ; m_{3}=5.5+0.01 i ;$ (d) $r_{1}=30.0 \mathrm{~nm} ; h_{1}=7.5 \mathrm{~nm} ; h_{2}=22.5 \mathrm{~nm} ; m_{1}=5+0.1 i ; m_{2}=$ $4.5+0.1 i ; m_{3}=3.8+0.1 i$; (e) $r_{1}=30.0 \mathrm{~nm} ; h_{1}=8.5 \mathrm{~nm} ; h_{2}=20.0 \mathrm{~nm} ; m_{1}=5+0.1 i ; m_{2}=4.7+0.1 i$; $m_{3}=4.0+0.1 i ;$ (f) $r_{1}=30.0 \mathrm{~nm} ; h_{1}=8.5 \mathrm{~nm} ; h_{2}=18.5 \mathrm{~nm} ; m_{1}=5+0.1 i ; m_{2}=4.8+0.1 i ; m_{3}=4.5+0.1 i$. The refractive index of the host material was set to 1 ; the filling fraction was $\sim 0.30$ for $\mathrm{THz}$ and $\sim 0.39$ for visible range.

As mentioned above, the effective permittivity and permeability of the material were calculated using the field-averaging technique [55]. The most common method for extracting the effective material parameters is based on the analysis of the scattering parameters. The comparison of the S-parameters calculated with the effective permittivity and permeability with those obtained by direct simulation is shown in Fig. 7. The S-parameters were calculated for the transmission of an electromagnetic wave through a slab made of three layers of particles. It can be seen that, even for such a thin slab, the homogenized effective parameters produce a fairly good agreement.
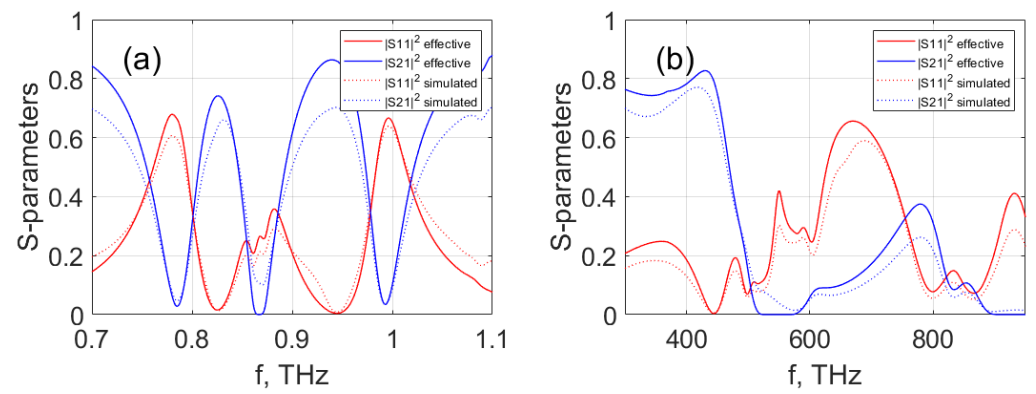

Fig. 7. Comparison of transmission (red) and reflection (blue) coefficients for the effective parameters of the slab of metamaterial (full line) and direct simulations (dotted line) in the $\mathrm{THz}$ (a) and visible (b) frequency ranges. 
The visualization of transmission through a slab of the proposed metamaterial demonstrating the evident left-handed behavior are available in the Supplementary Information.

The above results indicate that both negative permittivity and negative permeability can be simultaneously achieved by the special design of all-dielectric meta-atoms. The particular property of the proposed design is its isotropy, which allows macroscopic metamaterials to be produced in bulk without any special spatial arrangement. For instance, the proposed metaatoms might be suspended in a low-refractive index liquid (e.g, water with refractive index of 1.33 at visible band or transformer oil with refractive index of $\sim 1.5$ at $\mathrm{THz}$ ), which would still provide negative refraction as far as the filling fraction is sufficient. Alternatively, such a material might be immersed in a liquid crystal, which could offer infinite possibilities of additional tunability by external factors such as temperature, electric or magnetic field, and optical nonlinearity.

\section{Conclusion}

In this work, we have proposed the design of isotropic all-dielectric meta-atoms, which simultaneously provide negative permittivity and negative permeability. The proposed design is based on specially adapted layer properties of three-layer spherical particles. The isotropy of the particles suggests the possibility of using them in a negative-refraction liquid metamaterial, because no special arrangement is needed for such particles to exhibit the double-negative behavior. Thus, the proposed meta-atoms may be suspended in a lowrefractive index fluid or in a nematic liquid crystal, forming a highly tunable liquid doublenegative metamaterial. In the current study, first, the layer parameters were optimized within the framework of the Mie scattering theory and the MG homogenization method. It has been shown that, when the layer parameters are within a certain area of the parametric space, an ensemble of such particles can exhibit double-negative behavior in a wide range of frequencies. The feasibility of the proposed design was then verified using numerical simulations. The simulation results confirm the double-negative behavior of the medium composed of such particles, for instance in the $\mathrm{THz}$ and visible frequency ranges. In addition, the sensitivity of the proposed design to variations in the layer parameters was studied. It has been shown that a slight deviation from the optimal parameters does not lead to a break in the double-negative behavior. Finally, the effective medium approach was compared with direct simulations applied to the transmission through a metamaterial slab.

\section{Funding}

This study was supported by the French PIA project "Lorraine Université d'Excellence", reference ANR-15-IDEX-04-LUE and TALiSMAN project, funded by FEDER (2019000214).

\section{Disclosures}

The authors declare no conflicts of interest.

\section{References}

1. V.G. Veselago, "Electrodynamics of substrates with simultaneously negative electrical and magnetic permeabilities", Sov. Phys. Usp. 10, 5-13 (1968).

2. J. Pendry, A. Holden, W. Stewart, and I. Youngs, "Extremely Low Frequency Plasmons in Metallic Mesostructures," Phys. Rev. Lett. 76, 4773-4776 (1996).

3. J. B. Pendry, A. J. Holden, D. J. Robbins, and W. J. Stewart, "Magnetism from conductors and enhanced nonlinear phenomena," IEEE Trans. Microw. Theory Tech. 47 (1999).

4. D. R. Smith, W. J. Padilla, D. C. Vier, S. C. Nemat-Nasser, and S. Schultz, "Composite medium with simultaneously negative permeability and permittivity," Phys. Rev. Lett. 84, 4184-4187 (2000).

5. R. A. Shelby, D. R. Smith, and S. Schultz, "Experimental verification of a negative index of refraction," Science 292, 77-79 (2001).

6. S. Linden, C. Enkrich, M. Wegener, T. Zhou, T. Kochny, and C. M. Soukoulis, "Magnetic response of metamaterials at 100 terahertz," Science 306, 1351-1353 (2004). 
7. S. Zhang, W. Fan, B. K. Minhas, A. Frauenglass, K. J. Malloy, and S. R. J. Brueck, "Midinfrared resonant magnetic nanostructures exhibiting a negative permeability," Phys. Rev. Lett. 94, 037402 (2005).

8. G. Dolling, M. Wegener, C. M. Soukoulis, and S. Linden, "Negative-index metamaterial at $780 \mathrm{~nm}$ wavelength," Opt. Lett. 32, 53 (2007).

9. U. K. Chettiar, A. V. Kidishev, H. K. Yuan, W. Cai, S. Xiao, V. P. Drachev, and V. M. Shalaev, "Dual-band negative index metamaterial: double negative at $813 \mathrm{~nm}$ and single negative at $772 \mathrm{~nm}$," Opt. Lett. 32, 16711673 (2007)

10. H. J. Lezec, J. A. Dionne, and H. A. Atwater, "Negative refraction at visible frequencies," Science 316, 430432 (2007).

11. A. Minovich, J. Farnell, D. N. Neshev, I. McKervacher, F. Karouta, J. Tian, D. A. Powell, I. V. Shadrivov, H. H. Tan, C. Jagadish, and Y. S. Kivshar, "Liquid crystal based nonlinear fishnet metamaterials," Appl. Phys. Lett. 100, 121113 (2012).

12. A. A. Zharov, I. V. Shadrivov, and Y. S. Kivshar, "Nonlinear properties of left-handed metamaterials," Phys. Rev. Lett. 91, 037401 (2003).

13. M. A. Noginov, Y. A. Barnakov, G. Zhu, T. Tumkur, H. Li, and E. E. Narimanov, "Bulk photonic metamaterial with hyperbolic dispersion,” Appl. Phys. Lett. 94, 151105 (2009).

14. Y. Guo, W. Newman, C. L. Cortes, and Z. Jacob, “Applications of hyperbolic metamaterial substrates,” Adv. Opt. Electron. 2012, 452502 (2012).

15. A. Poddubny, I. V. Iorsh, P. A. Belov, and Y. S. Kivshar, "Hyperbolic metamaterials," Nat. Photonics 7, 948957 (2013).

16. A. Alù, M. Silveirinha, A. Salandrino, and N. Engheta, "Epsilon-near-zero metamaterials and electromagnetic sources: Tailoring the radiation phase pattern,” Phys. Rev. B 75, 155410 (2007).

17. R. Liu, Q. Cheng, T. Hand, J. J. Mock, T. J. Cui, S. A. Cummer, and D. R. Smith, "Experimental demonstration of electromagnetic tunneling through an epsilon-near-zero metamaterial at microwave frequencies," Phys. Rev. Lett. 100, 023903 (2008).

18. Y. A. Urzhumov, G. Shvets, J. A. Fan, F. Capasso, D. Brand1, and P. Nordlander, "Plasmonic nanoclusters: a path towards negative-index metafluids," Opt. Express 15, 14129-14145 (2007).

19. M. Fruhnert, S. Muhlig, F. Lederer, and C. Rockstuhl, "Towards negative index self-assembled metamaterials," Phys. Rev. B 89, 075408 (2014).

20. A. A. Zharov, A. A. Zharov, Jr., and N. A. Zharova, "Liquid metacrystals," J. Opt. Soc. Am. B 31, 559 (2014).

21. R. Kowerdziej, J. Krupka, E. Nowinowski-Kruszelnicki, M. Olifierczuk, and J. Parka, "Microwave complex permittivity of voltage-tunable nematic liquid crystals measured in high resistivity silicon transducers," Appl. Phys. Lett. 102, 102904 (2013).

22. R. Kowerdziej, M. Olifierczuk, and J. Parka, "Thermally induced tunability of terahertz metamaterial by using a specially designed nematic liquid crystal mixture,” Opt. Express 26, 2443 (2018).

23. D. H. Werner, D.-H. Kwon, I.-C. Khoo, A. V. Kildishev, and V. M. Shalaev, "Liquid crystal clad near-infrared metamaterials with tunable negative-zero-positive refractive indices," Opt. Express 15, 3342 (2007).

24. D. Schurig, J. J. Mock, B. J. Justice, S. A. Cummer, J. B. Pendry, A. F. Starr, and D. R. Smith, "Metamaterial electromagnetic cloak at microwave frequencies," Science 314, 977 (2006).

25. B. Edwards, A. Alu, M. G. Silveirinha, and N. Engheta, "Experimental verification of plasmonic cloaking at microwave frequencies with metamaterials," Phys. Rev. Lett. 103, 153901 (2009).

26. J. B. Pendry, "Negative Refraction Makes a Perfect Lens," Phys. Rev. Lett. 85, 3966 (2000).

27. P. A. Belov, Y. Hao, and S. Sudhakaran, "Subwavelength microwave imaging using an array of parallel conducting wires as a lens," Phys. Rev. B 73, 033108 (2006)

28. J. B. Pendry, D. Schurig and D.R. Smith, "Controlling Electromagnetic Fields," Science 312, 1780 (2006).

29. U. Leonhardt, “Optical Conformal Mapping," Science 312, 1777 (2006).

30. F. Zhang, C. Li, Y. Fan, R. Yang, N.- H. Shen, Q. Fu, W. Zhang, Q. Zhao, J. Zhou, T. Koschny, and C. M. Soukoulis, "Phase- Modulated Scattering Manipulation for Exterior Cloaking in Metal-Dielectric Hybrid Metamaterials,” Adv. Mater. 31, 1903206 (2019).

31. W. Zhu, R. Yang, Y. Fan, Q. Fu, H. Wu, P. Zhang, N.-H. Shen, and F. Zhang, "Controlling optical polarization conversion with $\mathrm{Ge}_{2} \mathrm{Sb}_{2} \mathrm{Te}_{5}$-based phase-change dielectric metamaterials," Nanoscale 10, 12054 (2018).

32. Y. Tang and A. E. Cohen, "Optical Chirality and Its Interaction with Matter," Phys. Rev. Lett. 104, 163901 (2010).

33. E. Plum, V. A. Fedotov, and N. I. Zheludev, "Optical activity in extrinsically chiral metamaterial," Appl. Phys. Lett. 93, 191911 (2008).

34. A. Andryieuski, C. Menzel, C. Rockstuhl, R. Malureanu, and A. V. Lavrinenko, "The split cube in a cage: Bulk negative-index material for infrared applications," J. Opt. A 11, 114010 (2009).

35. O. Paul, C. Imhof, B. Reinhard, R. Zengerle, and R. Beigang, "Negative index bulk metamaterial at terahertz frequencies," Opt. Express 16, 6736 (2008).

36. S. Xiao, U. K. Chettiar, A. V. Kildishev, V. P. Drachev, and V. M. Shalaev, "Yellow-light negative-index metamaterials," Opt. Lett. 34, 3478 (2009).

37. P. Weis, O. Paul, C. Imhof, R. Beigang, and M. Rahm, "Strongly birefringent metamaterials as negative index terahertz wave plates," Appl. Phys. Lett. 95, 171104 (2009).

38. P. Ding, E. J. Liang, W. Q. Hu, L. Zhang, Q. Zhou, and Q. Z. Xue, "Numerical simulations of terahertz doublenegative metamaterial with isotropic-like ${ }^{-}$shnet structure," Photon. Nanostruct. Fund. Appl. 7, 92 (2009). 
39. J. Zhou, T. Koschny, M. Kafesaki, E. N. Economou, J. B. Pendry, and C. M. Soukoulis, "Saturation of the Magnetic Response of Split-Ring Resonators at Optical Frequencies,” Phys. Rev. Lett. 95, 223902 (2005).

40. S. Jahani, Z. Jacob, "All-dielectric metamaterials," Nature Nanotech 11, 23-36 (2016).

41. C. F. Bohren, D. R. Huffman, Absorption and Scattering of Light by Small Particles (John Wiley \& Sons, 2008).

42. R. Gomez-Medina, B. Garcia-Camara, I. Suarez-Lacalle, F. González, F. Moreno, M. Nieto-Vesperinas, and J. J. Saenz, "Electric and magnetic dipolar response of germanium nanospheres: interference effects, scattering anisotropy, and optical forces," J. Nanophoton. 5, 053512 (2011).

43. A. García-Etxarri, R. Gómez-Medina, L. S. Froufe-Pérez, C. López, L. Chantada, F. Scheffold, J. Aizpurua, M. Nieto-Vesperinas, and J. J. Sáenz, "Strong magnetic response of submicron Silicon particles in the infrared," Opt. Express 19, 4815 (2011).

44. R. Paniagua-Domınguez, F. Lopez-Tejeira, R. Marques, and J. A. Sanchez-Gil, "Metallo-dielectric core-shell nanospheres as building blocks for optical three-dimensional isotropic negative-index metamaterials," New J. Phys. 13, 123017 (2011).

45. M. S. Wheeler, J. S. Aitchison, and M. Mojahedi, "Coated nonmagnetic spheres with a negative index of refraction at infrared frequencies," Phys. Rev. B 73, 045105 (2006).

46. V. Yannopapas, "Negative refractive index in the near-UV from Au-coated CuCl nanoparticle superlattices," Phys. Stat. Sol. (RRL) 1, 208 (2007).

47. X. Huang, Y. Lai, Z. H. Hang, H. Zheng, and C. T. Chan, "Dirac cones induced by accidental degeneracy in photonic crystals and zero-refractive-index materials," Nature Mater. 10, 582-586 (2011).

48. I. B. Vendik, O. G. Vendik, I. A. Kolmakov, and M. P. Odit, "Modelling of isotropic double negative media for microwave applications," Opt. Electron. Rev. 14, 179-186 (2006).

49. S. Townsend, S. Zhou, and Qing Li, "Double-negative metamaterial from conducting spheres with a highpermittivity shell," Opt. Lett. 39, 4587-4590 (2014).

50. E. F. Kuester, N. Memic, S. Shen, A. D. Scher, S. Kim, K. Kumley, and H. Loui, "A negative refractive index metamaterial based on a cubic array of layered nonmagnetic spherical particles," Progress In Electromagnetics Research B 33(33), 175 (2011).

51. Q. Duan, S. Li, and B. Hou, “A microsphere suspension model of metamaterial fluids,” AIP Advances 7, 055015 (2017).

52. J. R. Wait, "Electromagnetic scattering from a radially inhomogeneous sphere," Applied Scientific Research B 10, 441-450 (1963).

53. O. Peña and U. Pal, "Scattering of electromagnetic radiation by a multilayered sphere," Computer Physics Communications 180, 2348-2354 (2009).

54. W. T. Doyle, "Optical properties of a suspension of metal spheres," Phys. Rev. B 39, 9852 (1989).

55. J. D. Jackson, Classical Electrodynamics, 3rd ed., Wiley, New York, 1999.

56. C. Dupas, S. Guillemet-Fritsch, P. Geffroy et al., "High permittivity processed SrTiO3 for metamaterials applications at terahertz frequencies," Sci Rep 8, 15275 (2018).

57. D. R. Smith and J. B. Pendry, "Homogenization of metamaterials by field averaging (invited paper)," J. Opt. Soc. Am. B 23, 391-403 (2006) 